\title{
Quality Control of Obstetric and Gynecologic Ultrasound in Hungary: Education and Expectations
}

Zoran Belics, Zoltán Papp

\begin{abstract}
The result of ultrasound examinations depends heavily on the preparation of the personnel carrying out the examination and the technical capabilities of the equipment they use. Only wellorganized regional or national programs are able to provide high level, cost-effective care based on certification of quality. Such certification must include the training of professionals, the definition of competence levels, accreditation of laboratories and the establishment of professional protocols. The ultrasound examinations in Hungary are performed by obstetricians under the recommendation of the Hungarian Society of Ultrasound in Obstetrics and Gynecology and there are well defined levels and protocols of attendance. The different levels of attendance require proficiency at different levels and this requires regular training which is assured by the Society and gives a concern for all doctors and sonographers who perform ultrasound examinations and for all patients who receive them. The well organized quality assurance in obstetric and gynecologic ultrasound examinations is an essential dimension of high quality obstetric and gynecologic medical attendance.
\end{abstract}

Keywords: Ultrasound in obstetrics and gynecology, Quality assurance, Hungarian model, Competence levels, Professional protocols, Accreditation of laboratories.

How to cite this article: Belics Z, Papp Z. Quality Control of Obstetric and Gynecologic Ultrasound in Hungary: Education and Expectations. Donald School J Ultrasound Obstet Gynecol 2013;7(4):492-495.

\section{Source of support: Nil}

Conflict of interest: None declared

\section{INTRODUCTION}

The use of ultrasound has revolutionized medicine, including obstetrics and gynecology in the past four decades. It constitutes the cornerstone of modern obstetric care and gynecologic practice (Figs 1 and 2). A mong the available noninvasive imaging examinations, ultrasound is the most common one in which ionizing radiation is not involved.

Ultrasound examinations were introduced into medicine in the 1970s. A t that time, equipment provided static images. During the 1980s technology developed rapidly and, with the appearance of real-time machines, this method of imaging became indispensable for obstetricians and gynecologists. By the 1990s, ultrasound examination had become accessible for almost everyone in developed countries. The three-dimensional (Figs 3 and 4) and realtime 3D technologies opened new horizons for examination and interpretation of intrauterine images by the end of the 1990s. $^{1}$
Ultrasound is widely used for the prenatal evaluation of fetal anatomy (Figs 5 and 6 ) and growth as well as for the diagnosis and follow-up of gynecologic disorders (Fig. 7). Moreover, the ultrasound procedure is relatively easy, misuse by physicians without adequate training is possible which, unfortunately, and common produce missed diagnoses of gross abnormalities and incorrect treatment of patients. ${ }^{2}$ From the other side, the patient is intensely involved in the ultrasound examination and has the right to know the level of qualification of the physician who perform the ul trasound scan and the board level of institution where the scan is performed.

\section{HUNGARIAN EXPERIENCES}

In Hungary, traditionally nearly $100 \%$ of the physicians who perform obstetric and gynecologic ultrasound scans are obstetricians and gynecologists, who are very knowledgeable in fetal anatomy and physiology as well as in anatomy and physiology of the internal female genital organs..$^{3-5}$ Hungary was one of the first countries to introduce the routine screening of the pregnant population. ${ }^{6}$ It is worth carrying out routine screening in each pregnancy because the majority of abnormalities occur in pregnancies with low risk. Screened cases and the examination of high risk groups have to be referred to higher level centers. Here, appropriate technical background and qualified personnel are present

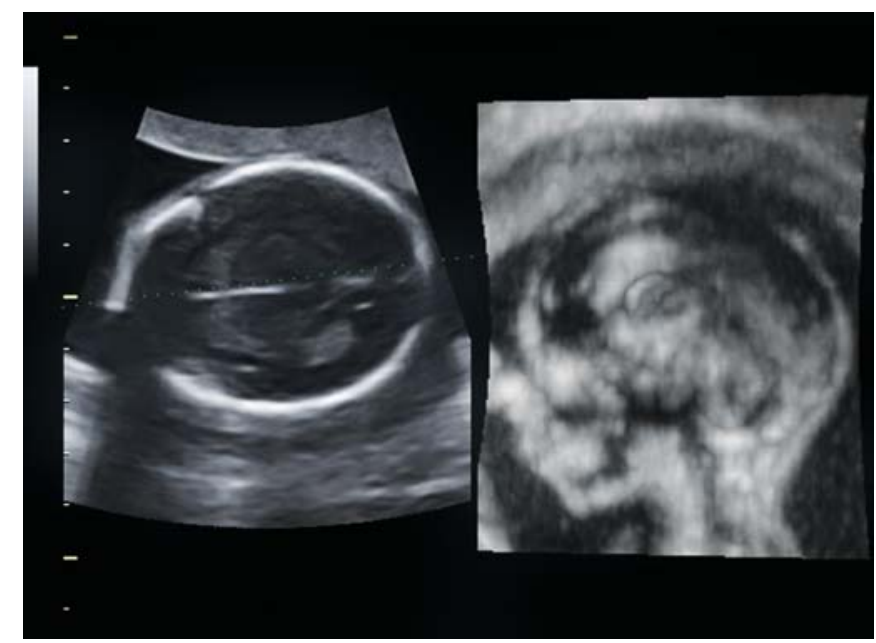

Fig. 1: Volume contrast imaging ( $\mathrm{VCl})$ in the C-plane: in the axial scan a line is selected passing through the cavum septi pellucidi between the two hemispheres. The sagittal view (C-plane) orthogonal the axial one is simultaneously displaced showing the corpus callosum (19 weeks of gestation) 


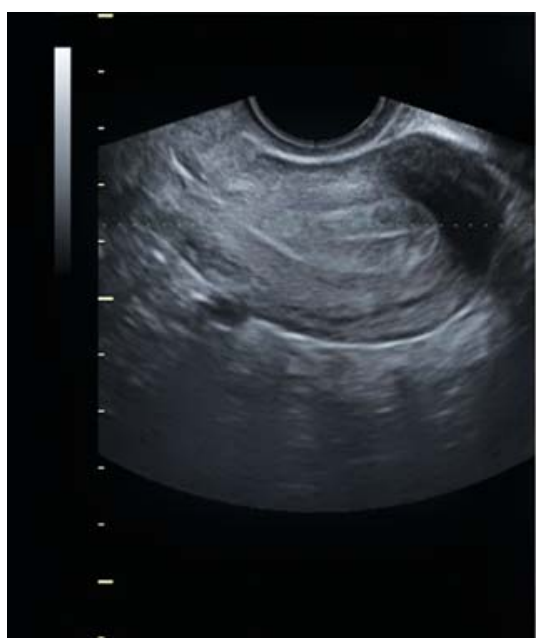

Fig. 2: A coronal view of the uterus

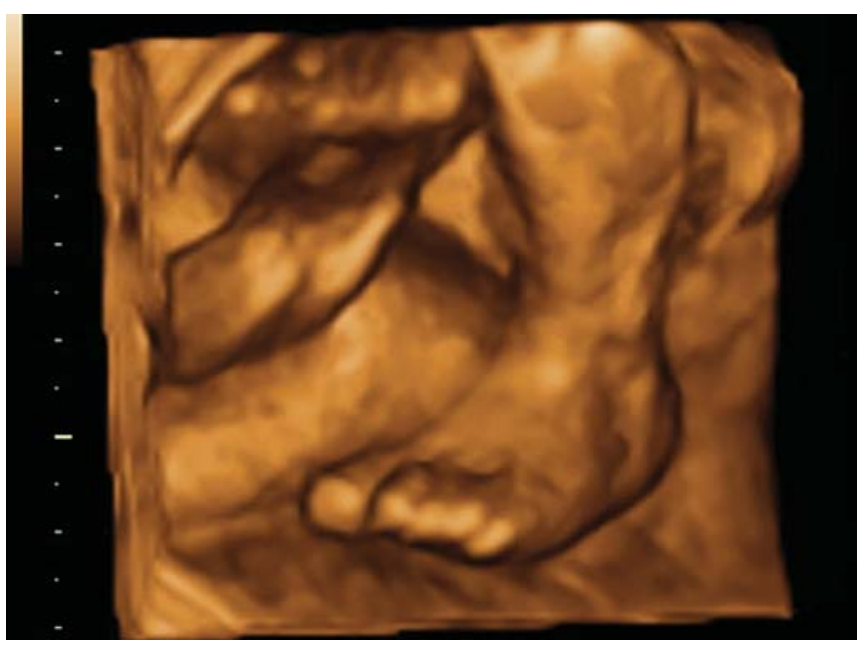

Fig. 4: 3D image of the fetal foot (24 weeks of gestation)

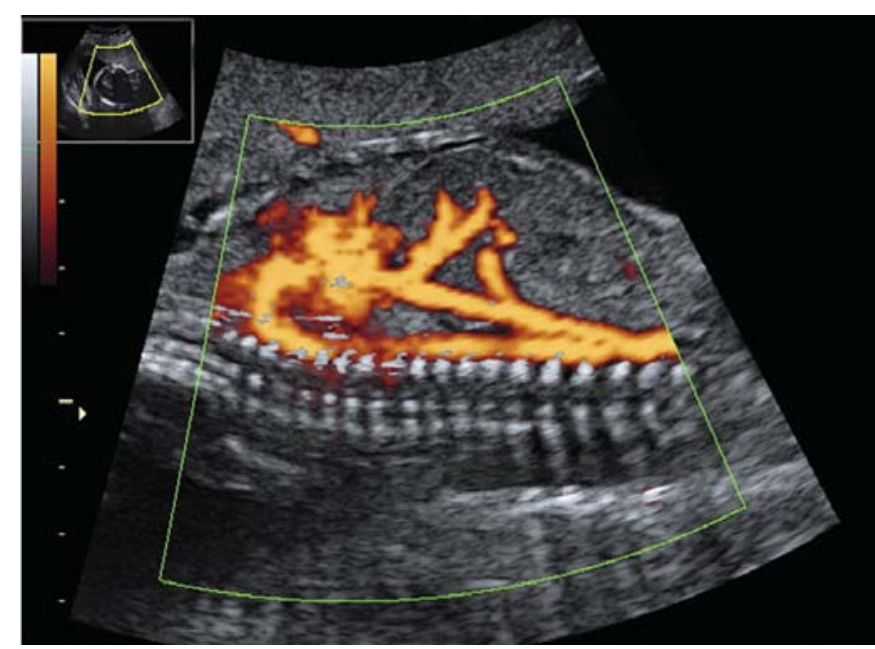

Fig. 6: Sagittal view of the fetal thorax: aortic arch, inferior cava vein (22 weeks of gestation)

to provide cost-effective care. At the same time, necessary invasive interventions can also be performed.

It is therefore essential that obstetricians and gynecologists, as well as sonographers are properly trained

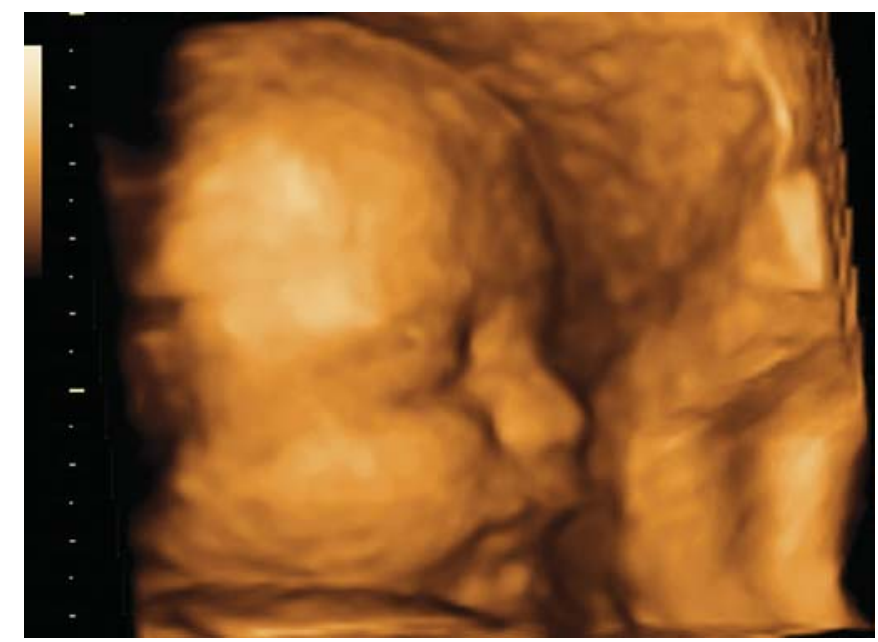

Fig. 3: 3D image of fetal face (28 weeks of gestation)

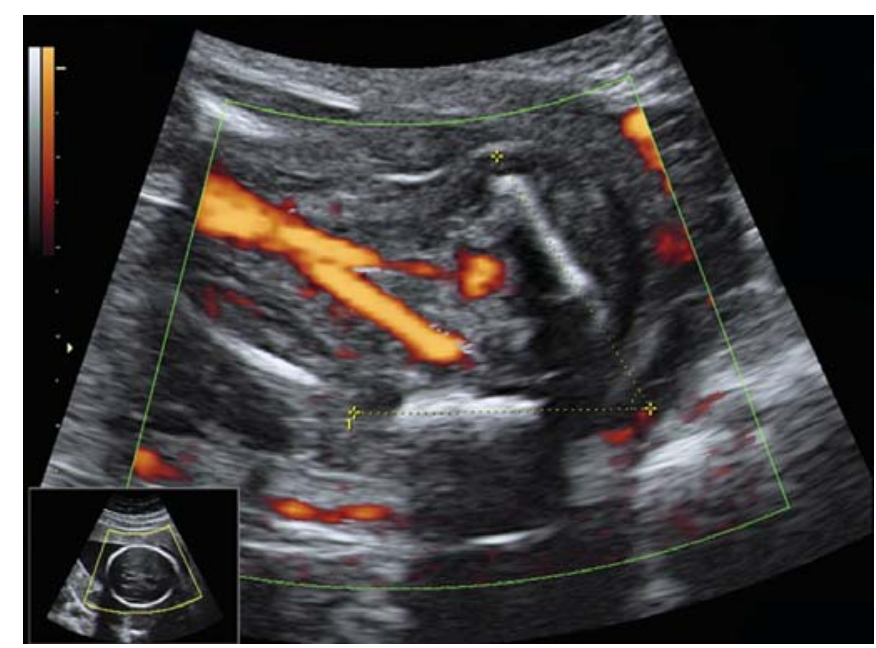

Fig. 5: Transverse section of the fetal pelvis (20 weeks of gestation): iliac wing angle, umbilical arteries

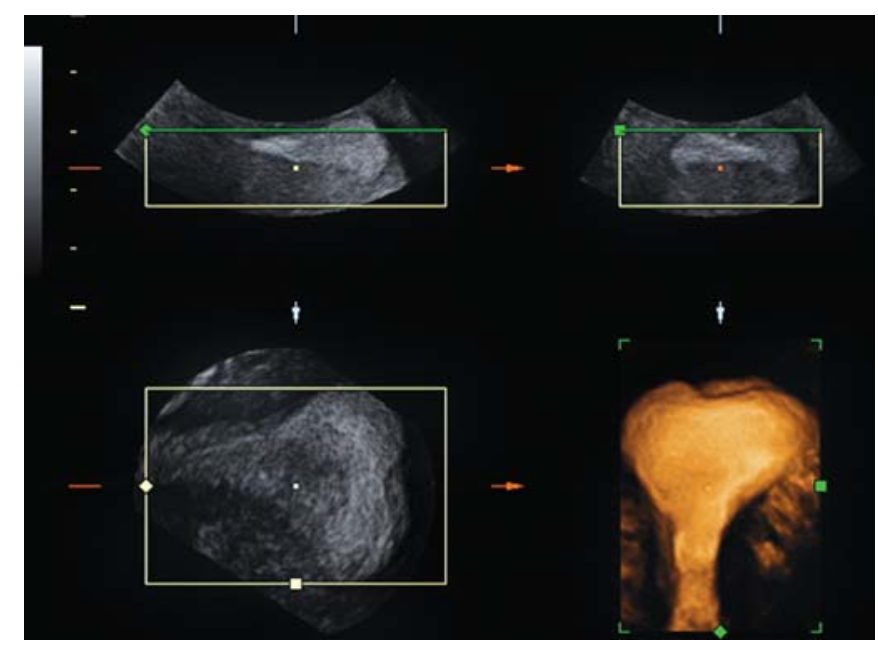

Fig. 7: Coronal view of the uterus: uterus arcuatus

and protocol of ultrasound examination is established. In order to assure the quality of ultrasound examinations, the Hungarian Society of UItrasound in Obstetrics and Gynecology (M agyar Szülészeti és Nõgyógyászati 
Ultrahang Társaság - M SZN UT) was organized in 1992. 7,8 Recently this society is the biggest organization for obstetricians and gynecologists in Hungary, which has more than 1,400 members.

The main objectives of the Society are:

- Take a stand on guidelines in obstetrical and gynecological ultrasound procedures and coordination of these guidelines at the national level

- Take a part in the gradual and postgradual educations

- Help the theoretical and practical provision

- Give countenance for professional activities of its members and supervise the level of the professional quality

- Give proficiency certificates, with which confirms the readiness of individuals and protect them against unfounded attacks.

The Hungarian Society of Ultrasound in Obstetrics and Gynecology performs an important educational role, which helps assure quality. It directly assures quality by making regular evaluations of the quality and the condition of equipment.

The Society organizes four postgraduate courses every year, with an average of 100 participants, and holds the Society's N ational Congress every 2 years for almost 1,000 registered participants. The participation on organized courses and congresses are essential requirement for renewal.

\section{LEVELS OF PROFICIENCY CERTIFICATES}

There are three different levels of proficiency certificates, which are given by the Society:

- $\operatorname{Basic}(A)$,

- Intermediate (B) and

- Specialist (C).

Each level requires a medical undergraduate degree. The main subject to receive the certificate is board exam and adequate number of performed examinations, which are permitted by the head of the accredited ultrasound unit, as well as the passed time in ultrasound unit and scientific work (depend from the level). The certificates are valid for 1 year. Every year the practitioner must renewed certificate with evidence of passing a special examination at the appropriate level and completing the required training.

The Society has developed the minimal training requirements for the three levels of proficiency certificates. Basic ' $A$ ' level requires postgraduate course followed by theoretical and practical examination, 3 months of training (400 obstetric and 200 gynecologic ultrasound examinations) under strict supervision. For intermediate ' $B$ ' level two postgraduate courses followed by theoretical and practical examination, 5 years of training $(5,000$ obstetric and 2,000 gynecologic scans) are required. Specialist ' $C$ ' level is highest level of proficiency. For this level there must be evidence of academic productivity, including at least one presentation per year at advanced courses or congresses. Furthermore, the specialist needs specialized training in a tertiary center.

Behind the doctors there are sonographers, who are specially trained midwifes with completed theoretical educations and examinations in obstetric and gynecologic ultrasound examinations (level of university education). The special practical training is also required. The competence of sonographers is similar with the competence of basic 'A' level (first and third trimester scan, gynecologic ultrasound examination).

In H ungary, the second trimester ultrasound examination performed between 18 and 22 weeks of gestation are limited to those practitioners with intermediate or specialist (' $B$ ' and ' $C$ ') levels as well as the specialized gynecologic invasive procedures. However, for the first trimester scan, the certificate of Fetal Medicine Foundation (FMF) is required.

\section{OBJECTIVE CONDITIONS, BOARD LEVELS}

Likewise to examiners, in Hungary, there are three board levels, ranged from basic to upper levels, which are accredited by the Society. To receive the accreditation every ultrasound laboratory has to fulfill requirements based on skill as well as equipment and circumstances.

\section{Basic Level}

Screening or diagnostic ultrasound examination performed in private practice, practitioners, city hospital. In the case of a suspect or pathological case the requisition of superior level consul tation is required. Examinations are performed by specialists with ' $A$ ', ' $B$ ' and ' $C$ ' proficiency certificates or sonographers.

\section{Intermediate Level}

'High risk' population screening or examination of the previously screened pathological cases in regional center. The diagnosis or differential diagnoses of the abnormal cases, Doppler examinations are expected on this level. If the special examination (fetal echocardiography, genetic counseling, biochemistry) is needed, higher level consultation is required. Examinations are performed by specialists with ' $\mathrm{B}$ ' and ' $\mathrm{C}$ ' proficiency certificates. 


\section{Upper Level (Special Supply)}

Final estimation of the pathological cases in prenatal diagnostic center accredited for ultrasound guided invasive procedure, public institution or institution appointed by the Society. Examinations are performed by specialists with ' $C$ ' proficiency certificates.

\section{TECHNICAL BACKGROUND AND EXPECTATION}

Fine resolution scanning, 2D real-time imagery, 3.5 to 5.0 M Hz convex, transabdominal phased vector array transducer and preferably 5-6.5 to 7.5-9 M Hz convex, sector, phased array transvaginal transducer is required for basic level. The availability for distance and circumference measurement is essential as well as the adequate way of the scan documentation.

Intermediate and upper level: high resolution scanning, 2D or 3/4D imagery, Doppler technique, 3.5 to $5.0 \mathrm{M} \mathrm{Hz}$ convex, transabdominal phased vector array transducer and preferably 5-6.5 to 7.5-9 M Hz convex, sector, phased array transvaginal transducer.

\section{LEGAL RELATIONS}

Unfortunately professional mistakes often are picked up by the press and reported sensationally. Because the number of legal firms specializing in such cases is increasing, it is now clear in medicine that is not enough to act correctly, but to document that you have done so. W ithout appropriate professional knowledge, the examiner performing the ultrasound examination gives an inaccurate report, find abnormalities that do not exist or, does worse yet, does not recognize existing abnormalities. An examination carried out in vain without proper indication, is harmless to the patient, but ties up professionals and equipment, thus decreasing accessibility for patients who actually need an ultrasound examination. ${ }^{1}$
A ppropriate technical background, qualified personnel and well-organized programs can guarantee the standard of care and provide legal protection for practitioners.

\section{REFERENCES}

1. Papp Z, Fekete T. The evolving role of ultrasound in obstetrics /gynecology practice. Int J Gynecol Obstet 2003 Sep;82(3): 339-346.

2. Foulkes $S$, J oubert $G, F$ aber $B L$, Hiemstra LA. Obstetrical ultrasound training of and practice by general practitioners in the private sector. Fam Pract $2004 \mathrm{~J} \mathrm{ul;46(6):25-27.}$

3. Tóth Z, C sécsei $K$, Szeifert GT, Papp Z. Prenatal diagnosis by ultrasound of midface defects associated with holoprosencephaly. A cta Chirurg Hung 1988;29(3):215-221.

4. Papp Z, C sécsei $K$, L indenbaum R H, Szeifert GT, Tóth Z, V áradi $\mathrm{V}$. A tlas of Fetal Diagnosis. A msterdam-L ondon-N ew $\mathrm{Y}$ orkTokyo: Elsevier; 1992 p.1-255.

5. Belics Z, Beke A, Csabay L, Szabó I, Papp Z. Sonographic measurement of the fetal iliac angle in trisomy 21,18 and 13. Fetal Diagn Ther 2003 J an-Feb; 18(1):47-50.

6. Papp Z, Tóth-Pál E, Papp C s, Tóth Z, Szabó M , V eress L, Török 0 . Impact of prenatal mid-trimester screening on the preval ence of fetal structural anomalies: a prospective epidemiological study. Ultrasound Obstet Gynecol 1995 Nov;6(5):320-326.

7. Papp Z. Quality assurance in obstetric and gynecological ultrasound in Hungary. Ultrasound Obstet Gynecol 1996 M ay; 7(5):305-306.

8. Szabó I, C sabay L, Tóth Z, Török O, Papp Z. Quality assurance in obstetric and gynecologic ultrasound. The H ungarian model. A nn NY A cad Sci 1998 J un;847:99-102.

\section{ABOUT THE AUTHORS}

\section{Zoran Belics}

Chief Consultant, Department of Obstetrics and Gynecology, Jahn Ferenc Hospital, H-1204 Budapest, K öves út 1, Budapest, Hungary

\section{Zoltán Papp (Corresponding Author)}

Professor and Chairman, Gynecology and Clinical Genetics, M aternity Private Hospital for Obstetrics, H-1125 B udapest, Kútvölgyi út4, Budapest Hungary, Phone: +36-206632366, e-mail: maternity@ maternity.hu 